EPJ Web of Conferences 59, 02017 (2013)

DOI: $10.1051 /$ epjconf/20135902017

(C) Owned by the authors, published by EDP Sciences, 2013

\title{
Symmetry issues in Directly Irradiated Targets
}

\author{
R. Ramis ${ }^{1, a}$, M. Temporal ${ }^{1}$, B. Canaud ${ }^{2}$ and V. Brandon ${ }^{2}$ \\ ${ }^{1}$ E.T.S.I. Aeronáuticos, Universidad Politécnica de Madrid, Spain \\ 2 CEA, DAM, DIF, 91297 Arpajon, France
}

\begin{abstract}
In direct drive Inertial Confinement Fusion (ICF), the typical laser beam to laser beam angle is around $30^{\circ}$. This fact makes the study of the irradiation symmetry a genuine $3 \mathrm{D}$ problem. In this paper we use the three dimensional version of the MULTI hydrocode to assess the symmetry of such ICF implosions. More specifically, we study a shock-ignition proposal for the Laser-Mégajoule facility (LMJ) in which two of the equatorial beam cones are used to implode and precompress a spherical capsule (the "reference" capsule of HiPER project) made of $0.59 \mathrm{mg}$ of pure Deuterium-Tritium mixture. The symmetry of this scheme is analysed and optimized to get a design inside the operating limits of LMJ. The studied configuration has been found essentially axial-symmetric, so that the use of 2D hydrocodes would be appropriate for this specific situation.
\end{abstract}

\section{INTRODUCTION}

To guarantee appropriate implosion symmetry, the current inertial laser fusion facilities have several hundred of laser beam lines, typically grouped in bundles ("quads") of four lines each. Neglecting any beam imperfections, the uniformity of irradiation depends on the number of irradiation directions (quads) rather than the absolute number of beams. This number ranges from 48 at the National Ignition Facility (NIF-USA) to 60 at Laser-Mégajoule (LMJ) [1]. These values can be effectively reduced if at a given instant of time only part of the beams are active. This occurs in when "zooming" or "shock ignition" schemes are used. In these conditions the typical quad-to-quad angle is around $30^{\circ}$, and this makes the analysis of the symmetry a genuine 3D problem. Several numerical approaches have been used for these studies, with different level of complexity but complementary each other: geometric calculation of the illumination of the target surface [2], ray tracing beam trajectories over 1D time dependent profiles [3], 2D [4], and 3D self consistent hydrodynamic simulations. In this paper we use the three dimensional version of the MULTI hydrocode [5] that includes 3D Lagrangian hydrodynamics with non-structured grids composed of tetrahedral, prismatic, and hexahedral cells, tabulated EOS, classical electronic heat conduction, and 3D laser ray tracing with refraction and absorption by inverse bremsstrahlung.

\section{SHOCK IGNITION AT LMJ}

Here, we will present the symmetry analysis of a shock-ignition proposal $[6,7]$ for the LMJ facility. In this facility, laser beam are organized in six cones around the vertical axis of the chamber, and the proposal considers to use two or four cones to irradiate directly a spherical capsule. Once the fuel has been compressed, short laser pulses from the two polar cones will produce a converging shock wave

\footnotetext{
ae-mail: rafael.ramis@upm.es
}

This is an Open Access article distributed under the terms of the Creative Commons Attribution License 2.0, which permits unrestricted use, distribution, and reproduction in any medium, provided the original work is properly cited. 

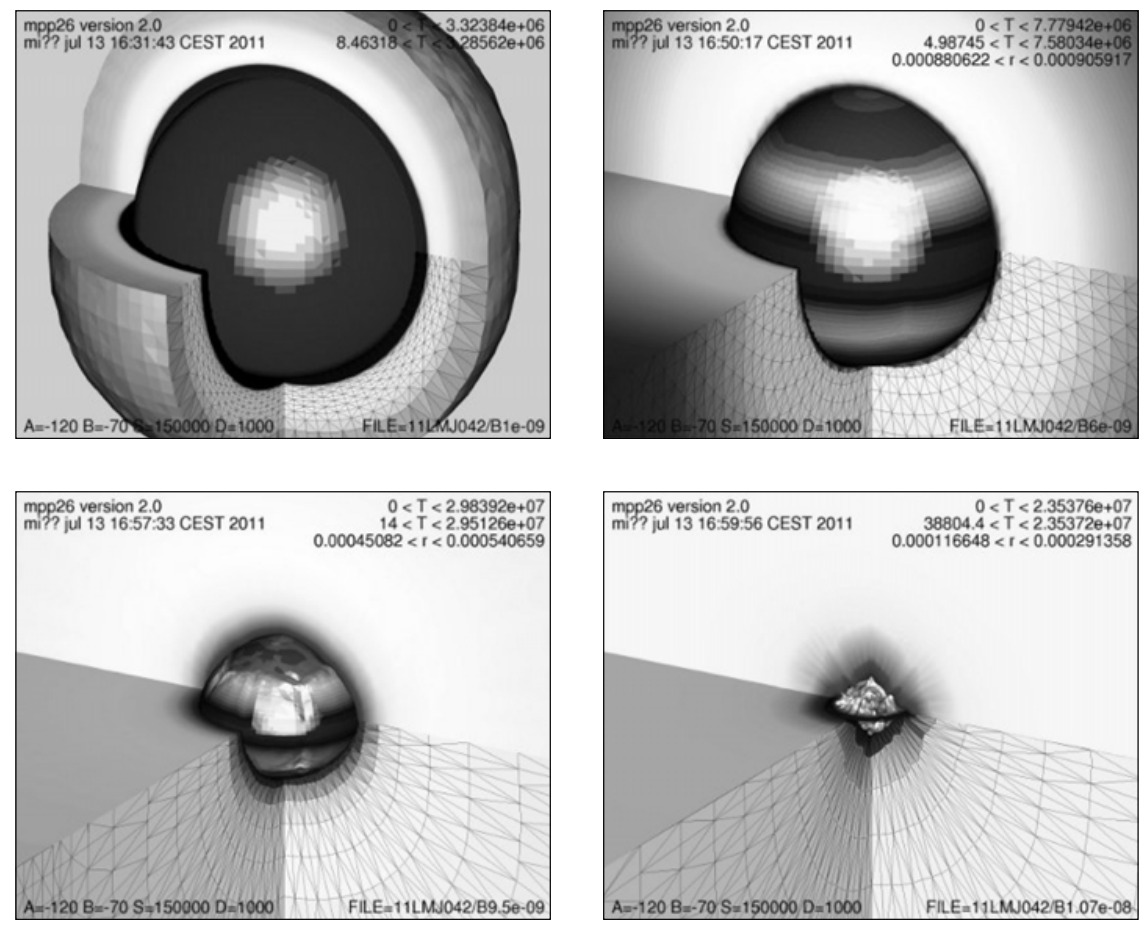

Figure 1. Representative MULTI-3D simulation results of an imploding capsule. Frames corresponds to different times: $1 \mathrm{~ns}, 6 \mathrm{~ns}, 9.5 \mathrm{~ns}$, and $10.7 \mathrm{~ns}$

that induces ignition. We take as a reference the capsule proposed in [8] which is a pure DeuteriumTritium (DT) capsule designed with a low entropy compression and to be ignited by external sources. This capsule is the reference one of the HiPER project [9], and has an internal radius of $833 \mu \mathrm{m}$ and a thickness of $211 \mu \mathrm{m}$ (fuel mass is $0.59 \mathrm{mg}$ ). The laser wavelength is $351 \mathrm{~nm}$ and the total absorbed laser energy is around $120 \mathrm{~kJ}$. A maximum fuel density of $650 \mathrm{~g} \mathrm{~cm}^{-3}$ is reached, with a maximum areal density of $\rho R=1.4 \mathrm{~g} \mathrm{~cm}^{-2}[10]$.

\section{THREE DIMENSIONAL SIMULATIONS}

Fig. 1 shows the configuration of a typical hydro simulation at four different times, showing the numerical grid and the temperature distribution in the expanding corona. The dark surface is a representative Lagrangian control surface fixed to the fluid that allows to visualize the distortion of the shell. Light and dark tonalities in the surface indicate the value of instantaneous radius below and above the average radius (overpressure and underpressure regions), respectively. After one nanosecond of irradiation, the plasma corona has been already created, and the ablation surface is clearly defined. At three nanoseconds, the first shock wave crosses the control surface, and the shell begins to implode. With 10 quads per cone (with $49^{\circ}$ half aperture), the implosion, at $6 \mathrm{ns,} \mathrm{appears} \mathrm{quite} \mathrm{uniform} \mathrm{in} \mathrm{azimuth,}$ giving place to a pronounced banded appearance in figure. At $9.5 \mathrm{~ns}$ the non-spherical shape of the control surface begins to be clearly visible. After $10.7 \mathrm{~ns}$, just before stagnation, the program stops due to the excessive grid distortion where very thin cells force a prohibitive reduction of the time step. As a representative magnitude defining the uniformity of the implosion we consider the "imploding 


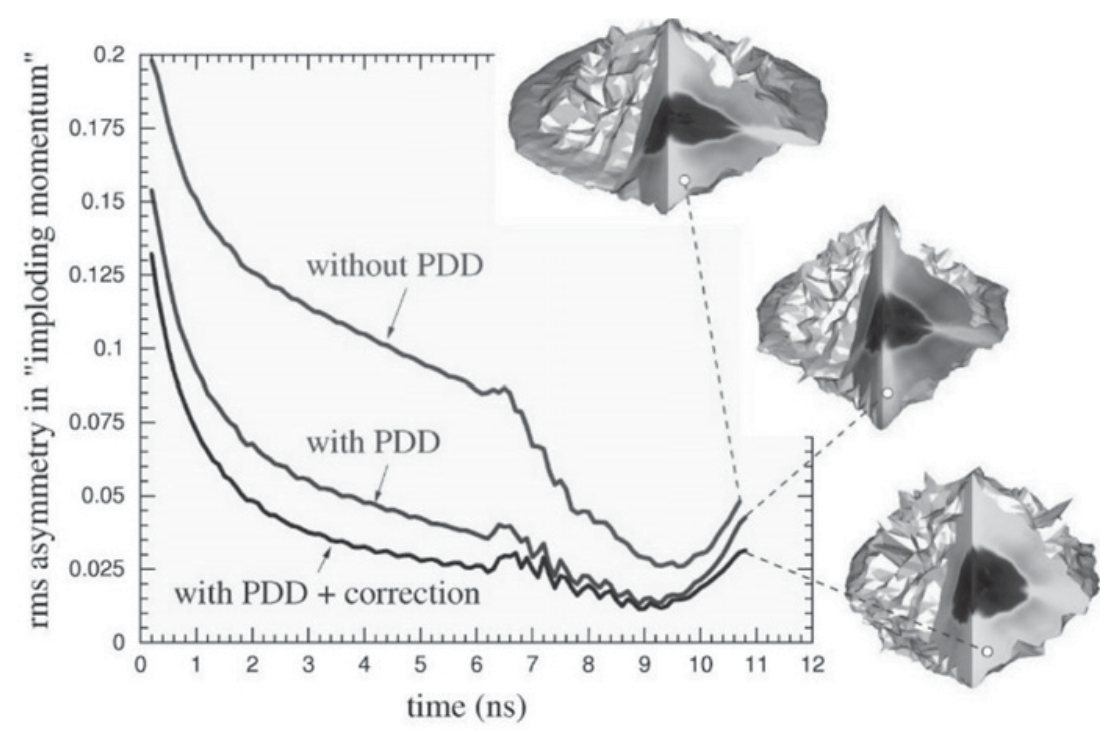

Figure 2. rms distortion for different irradiation schemes as a function of time. Shapes of the shell at the end of each simulation are also shown.

momentum" of the shell:

$$
p(\boldsymbol{\Omega}, t)=\int_{\boldsymbol{\Omega} \cdot \mathbf{v}<0} \boldsymbol{\Omega} \cdot \mathbf{v}(r, \boldsymbol{\Omega}, t) \rho(r, \boldsymbol{\Omega}, t) r^{2} d r,
$$

where the density $\rho$ and fluid velocity $\mathbf{v}$ are functions of time $t$, radial coordinate $r$, and direction $\boldsymbol{\Omega}$. At $6 \mathrm{~ns}$ the latitude variations of $p$ reach a root-mean-squared $(\mathrm{rms})$ value of $4.5 \%$ while the azimuthal variations are extremly small: $0.04 \%$. Only when a very reduced number of quads per cone are considered (4 quads, in an hypothetical configuration non-realizable at LMJ), the azimuthal variations become appreciable $(\simeq 0.3 \%)$. Fig. 2 shows the time dependent variation of the rms distortion in imploding momentum

$$
\sigma(t)=\frac{1}{\bar{p}(t)} \sqrt{\frac{1}{4 \pi} \int_{\mathbf{\Omega}}[p(\boldsymbol{\Omega}, t)-\bar{p}(t)]^{2} d \mathbf{\Omega}} \quad \text { with } \quad \bar{p}(t)=\frac{1}{4 \pi} \int_{\mathbf{\Omega}} p(\boldsymbol{\Omega}, t) d \mathbf{\Omega}
$$

for three selected cases. First we consider the situation where the beam axis cross the target centre. With this arrangement, the illumination model gives a quite poor symmetry (time independent) of around $11.5 \% \mathrm{rms}$, with high/low irradiation at the poles/equator of the capsule. Differently, the numerical value (curve "without PDD" in Fig. 2) depends of time. At the initial stages the numerical non-uniformity is worse (a factor 2) than in the illumination model. This is due to the fact that absorption, rather than illumination, determines the ablation pressure, and in the first stages of the process, absorption is fairly angle dependent. As time goes on, one can observe an improvement of the symmetry due to two factors not accounted in the illumination model: (a) as absorption efficiency increases, its dependence with incidence angle reduces, (b) light absorption takes place only at densities below critical density and some smoothing is induced by the diffusive transport of energy from the absorption region to the ablation surface. The combined effects reduce the value of $\sigma$ below 3\% during the implosion. At final stages (after $9.5 \mathrm{~ns}$ ) an increase of $\sigma$ is visible in Fig. 2. This later increment can be imputed to the hydrodynamic Rayleigh-Taylor instability that amplifies any distortion of the shell. 


\section{EPJ Web of Conferences}

\section{DESIGN IMPROVEMENTS}

One way to reduce the level of asymmetry is suggested by the predictions of the illumination model. It is known that displacing the beam axis a distance $\delta$ from target centre, the so called Polar Direct Drive (PDD) [11], the illumination symmetry can be improved. By varying the value of $\delta$, an optimum irradiation is obtained. For laser beams with intensity profile modelled by a supergaussian

$$
I(r)=I_{\max } \exp \left(-(r / \Delta)^{b}\right),
$$

the optimum illumination symmetry, with an rms distortion below one percent, is reached for $b=2.5$, $\delta=0.17 r_{o}$, and $\Delta=1.10 r_{0}$, where $r_{o}$ is the initial target radius. The corresponding three dimensional calculation shows a clear improvement (curve "with $P D D$ " in Fig. 2) with respect to the former configuration. However, the distortion $\sigma$ is always above the illumination prediction $(\simeq 1 \%)$. Now overpressure is located in the intermediate latitudes and the final core shape is slightly more spherical. Additional increments of $\delta$ spoil again the uniformity, because the overpressure moves from meridional regions to target equator. In principle one can improve the uniformity by trying all combinations of irradiation parameters $b, \delta$ and $\Delta$ to find an optimum that minimizes $\sigma$. Of course, the problem is the excessive required CPU time. A more practical strategy starts by quantifying the combined effect of all physical phenomena modifying the results of illumination model. At a selected time $t_{s}$, the asymmetry resulting from simulation can be adjusted by the results of the illumination model with beam parameters different to the ones fed into the simulation. This can be summarized as a variation of the "effective" values of beam parameters. For the previous case one finds that, for $t_{s}=6 \mathrm{~ns}$ (end of laser prepulse), $b_{e f f}=2.0$, and $\delta_{e f f}=0.12 r_{o}$ and $\Delta_{e f f}=0.99 r_{o}$. This drift can be compensated starting with modified laser parameters (e.g. if $\delta_{\text {eff }}$ is smaller that $\delta$, then we run again the code with a larger value of $\delta$, so that the resulting $\delta_{\text {eff }}$ would be closer to the desired value). With this strategy the uniformity can be improved as shown in Fig. 2 (curve "with PDD + correction"). The final configuration is clearly more spherical that in both previous calculations.

\section{SUMMARY AND ACKNOWLEDGEMENTS}

The 3D simulations allow us to evaluate the symmetry of irradiation schemes. Illumination calculations give only a crude description of the problem but supply useful hints of how to improve the results, so that optimization strategies can be developed combining both models. Although the present design has not been optimized from the energetic point of view, and the absorption efficiency is rather low (29\%), the compression pulse for HiPER class targets is inside the nominal bounds attainable by $2 \times 10$ quads of the LMJ. Requirements for the short laser pulse needed for shock ignition will depend on the symmetry reached in the compression phase [4], and work is under way to evaluate the corresponding power/energy threshold. Because the studied configuration has been found essentially axial-symmetric, the use of $2 \mathrm{D}$ hydrocodes is appropriate for such studies. This research has been supported by CICYT of Spain project ENE 2009 - 11668 and by the EURATOM association with CIEMAT.

\section{References}

[1] C. Cavailler, Plasma Phys. Control. Fusion 47, B389 (2005)

[2] A. J. Schmitt, Appl. Phys. Lett. 44, 399 (1984)

[3] M. Temporal, R. Ramis, and B. Canaud, J. Phy. Conf. Series 244, 022008 (2010)

[4] M. Temporal, R. Ramis, B. Canaud, V. Brandon, S. Laffite, and B. J. Le Garrec, Plasma Phys. Control. Fusion 53, 124008 (2011)

[5] R. Ramis, J. Meyer-ter-Vehn, and J. Ramírez, Comp. Phys. Comm. 180, 977 (2009) 


\section{IFSA 2011}

[6] B. Canaud, S. Laffite, V. Brandon, M. Temporal, and R.Ramis, Direct-drive shock ignition for the Laser MégaJoule, (these Proceedings)

[7] V. Brandon, B. Canaud, S. Laffite, M. Temporal, and R.Ramis, Systematic analysis of directdrive baseline designs for shock ignition with the Laser MégaJoule, (these Proceedings)

[8] S. Atzeni, A. Schiavi, and C. Bellei, Phys. Plasmas 14, 052702 (2007)

[9] M. Dunne, et al., HiPER Technical Background and Conceptual Design Report 2007, http://www.hiper-laser.org

[10] X. Ribeyre, M. Lafon, G. Schurtz, M. Olazabal-Loumé, J. Breil, S. Galera, and S. Weber, Plasma Phys. Control. Fusion 51, 124030 (2009)

[11] S. Skupsky et al., Phys. Plasmas 11, 2763 (2004) 